\title{
Prediction of China's Sulfur Dioxide Emissions by Discrete Grey Model with Fractional Order Generation Operators
}

\author{
Wei Meng (D), Daoli Yang $(\mathbb{D}$, and Hui Huang (i) \\ National Research Base of Intelligent Manufacturing Service, Chongqing Technology and Business University, Chongqing, China \\ Correspondence should be addressed to Wei Meng; mongvi@126.com
}

Received 20 May 2017; Accepted 8 November 2017; Published 29 January 2018

Academic Editor: Roberto Natella

Copyright (C) 2018 Wei Meng et al. This is an open access article distributed under the Creative Commons Attribution License, which permits unrestricted use, distribution, and reproduction in any medium, provided the original work is properly cited.

\begin{abstract}
Sulfur dioxide is an important source of atmospheric pollution. Many countries are developing policies to reduce sulfur dioxide emissions. In this paper, a novel prediction model is proposed, which could be used to forecast sulfur dioxide emissions. To improve the modeling procedure, fractional order accumulating generation operator and fractional order reducing generation operator are introduced. Based on fractional order operators, a discrete grey model with fractional operators is developed, which also makes use of genetic algorithms to optimize the modeling parameter $r$. The improved performance of the model is demonstrated via comparison studies with other grey models. The model is then used to predict China's sulfur dioxide emissions. The forecast result shows that the amount of sulfur dioxide emissions is steadily decreasing and the policies of sulfur dioxide reduction in China are effective. According to the current trend, by 2020, the value of China's sulfur dioxide emissions will be only $86.843 \%$ of emissions in 2015. Fractional order generation operators can be used to develop other fractional order system models.
\end{abstract}

\section{Introduction}

Sulfur dioxide (chemical formula $\mathrm{SO}_{2}$ ) is the most common form of sulfur oxides. The colorless gas has a strong irritating odor and is a major air pollutant. Inhaling it will lead to respiratory inflammation, bronchitis, emphysema, conjunctivitis, and other health problems. Sulfur dioxide will also weaken the immunity of young people, making them less resistant to infections. When sulfur dioxide reacts with water, sulfurous acid is formed [1]. If sulfurous acid is further oxidized by particulate matter in the atmosphere, sulfuric acid (the main component of acid rain) is rapidly and efficiently produced. With the presence of oxidants, light can catalyze the production of sulfate aerosols, which have an adverse effect on human health and could increase the mortality of patients [2]. Sulfur dioxide is the main cause of global "acid rain." It is estimated that the direct economic losses caused by metal corrosion in industrialized countries account for between 2 and 4 per cent of the gross domestic product (GDP). Due to the serious negative impact of sulfur dioxide on flora, fauna, and buildings, sulfur dioxide emissions and the emission concentrations are under strict regulation. The concentration of sulfur dioxide is an important indicator of whether the air is polluted. Using coal as the main energy source, China's sulfur dioxide emissions are ranked the first in the world [3]. Hence, it is of great practical significance to forecast the emissions of sulfur dioxide in order to formulate emission reduction policies.

The world has exerted great efforts to reduce sulfur dioxide emissions. There has also been a lot of research focusing on sulfur dioxide emissions. Lu and colleagues have studied sulfur dioxide emissions in China and sulfur trends in East Asia since 2000 [4] and also analyzed sulfur dioxide and primary carbonaceous aerosol emissions in China and India from 1996 to 2010 [5]. Smith et al. investigated global and regional anthropogenic sulfur dioxide emissions [6]. Ma et al. developed scenario analysis to study the reduction potential of sulfur dioxide emissions in China's iron and steel industry [7]. Yang and Hu conducted research on the inventory system of sulfur dioxide emissions in China [8]. Smith et al. discussed the methods and results of historical sulfur dioxide emissions from 1850 to 2000 [9]. Li et al. observed recent large reduction in sulfur dioxide emissions from China's power plants by using ozone monitoring instruments [10]. Hao et al. designated sulfur dioxide and acid rain pollution control zones 
and studied the impact on energy industry in China [11]. Mohajan studied China's sulfur dioxide emissions and local environmental pollution [12]. Zhou and Zhang established an improved metabolism grey model for predicting small samples with a singular datum and applied it to study sulfur dioxide emissions in China [13]. An adaptive neurofuzzy logic method has been developed by Yildirim et al. to estimate the impact of meteorological factors on sulfur dioxide pollution levels [14]. Hassanzadeh and coworkers developed statistical models and time series to forecast sulfur dioxide [15]. Li et al. looked into the contribution of China's emissions to global climate forcing [16]. Liu et al. believed that air pollutant emissions from Chinese households are a major and underappreciated source of ambient pollution [17].

To improve China's policies on sulfur dioxide emission reduction, it is necessary to predict China's future sulfur dioxide emissions. Grey prediction model is an important part of grey system theory $[18,19]$. It has been studied by many researchers and improved over the years [20-22]. Recently, Liu et al. [23], Wu et al. [24, 25], and Xiao et al. [26, 27] published results on fractional order accumulation and grey system model with the fractional order accumulation. However, all the existing research $[28,29]$ does not deduce the analytical expression of fractional order accumulating generation operator and fractional order reducing generation operator, nor does it prove that these fractional order generation operators satisfy mutual invertibility. Accumulating generation operator is similar to summation operator and reducing generation operator is similar to difference operator. Cheng and $\mathrm{Wu}[28,29]$ proposed the analytic expression of fractional summation operator but did not propose fractional difference operator and believed that the fractional difference operator generally does not satisfy the exponential rule. In this paper, both the fractional order accumulating generation operator and fractional order reducing generation operator are studied. Furthermore, a discrete grey model with fractional operators is used to predict China's future sulfur dioxide emissions.

The rest of the paper is organized as follows: in Section 2, the analytical expressions of fractional order accumulating generation operator and fractional order reducing generation operator are derived. In Section 3, the discrete grey model with fractional operators and order optimization algorithm is studied. This is followed by comparisons of the proposed model with the other discrete grey models, demonstrated via three case studies, in Section 4. In Section 5, the proposed discrete grey model with fractional operators is used to predict China's sulfur dioxide emissions. Then, conclusions are drawn in Section 6.

\section{Fractional Order Generation Operators}

Definition 1. Assume that

$$
X^{(0)}=\left(x^{(0)}(1), x^{(0)}(2), \ldots, x^{(0)}(n)\right)
$$

is a sequence of raw data and $D$ a sequence operator satisfying that

$$
X^{(0)} D=\left(x^{(0)}(1) d, x^{(0)}(2) d, \ldots, x^{(0)}(n) d\right),
$$

where

$$
x^{(0)}(k) d=\sum_{i=1}^{k} x^{(0)}(i), \quad k=1,2, \ldots, n
$$

and then the sequence operator $D$ is called a (first-order) accumulating generator of $X^{(0)}$, denoted as 1-AGO.

Definition 2. For $r \in \mathbf{Z}^{+}$, we define

$$
X^{(r)}=\left(x^{(r)}(1), x^{(r)}(2), \ldots, x^{(r)}(n)\right)
$$

as the $r$ th-order accumulating generation sequence of $X^{(0)}$, where

$$
\begin{array}{r}
x^{(r)}(k)=\sum_{i=1}^{k} x^{(r-1)}(i)=\sum_{i=1}^{k} \sum_{j=1}^{i} x^{(r-2)}(j), \\
\quad k=1,2, \ldots, n .
\end{array}
$$

Definition 3. Assume that the sequence of raw data is

$$
\begin{aligned}
& X^{(0)}=\left(x^{(0)}(1), x^{(0)}(2), \ldots, x^{(0)}(n)\right), \\
& X^{(1)}=\left(x^{(1)}(1), x^{(1)}(2), \ldots, x^{(1)}(n)\right)
\end{aligned}
$$

and is the first-order accumulating generation sequence of $X^{(0)}$, where

$$
x^{(1)}(k)=\sum_{i=1}^{k} x^{(0)}(i), \quad k=1,2, \ldots, n,
$$

and it follows that

$$
\begin{aligned}
X^{(1)} & =\left(x^{(0)}(1), x^{(0)}(1)\right. \\
& \left.+x^{(0)}(2), \ldots, \sum_{i=1}^{k} x^{(0)}(i), \ldots, \sum_{i=1}^{n} x^{(0)}(i)\right) .
\end{aligned}
$$

From Definition 2, it follows that

$$
x^{(r+1)}(k)=\sum_{i=1}^{k} x^{(r)}(i), \quad k=1,2, \ldots, n .
$$

So

$$
\begin{aligned}
X^{(2)} & =\left(x^{(1)}(1), x^{(1)}(1)\right. \\
& \left.+x^{(1)}(2), \ldots, \sum_{i=1}^{k} x^{(1)}(i), \ldots, \sum_{i=1}^{n} x^{(1)}(i)\right) \\
& =\left(x^{(0)}(1), 2 x^{(0)}(1)+x^{(0)}(2), \ldots, n x^{(0)}(1)\right. \\
& +(n-1) x^{(0)}(2)+\cdots+(n-k+1) x^{(0)}(k) \\
& \left.+x^{(0)}(n)\right) .
\end{aligned}
$$


Theorem 4. $X^{(2)}=\left(x^{(2)}(1), x^{(2)}(2), \ldots, x^{(2)}(n)\right)$ is the secondorder accumulating generation sequence of $X^{(0)}$, where

$$
x^{(2)}(k)=\sum_{i=1}^{k}(k+1-i) x^{(0)}(i) .
$$

Proof. By induction on $k$, consider the following.

First, for $k=1, x^{(2)}(1)=\sum_{i=1}^{k}(k+1-i) x^{(0)}(i)=x^{(0)}(1)$, so it is true.

Next, assume that, for some $k=s$ and $s \geq 1$, the statement is true. That is,

$$
x^{(2)}(s)=\sum_{i=1}^{s}(s+1-i) x^{(0)}(i) .
$$

Then, for $k=s+1$, it follows that

$$
\begin{aligned}
x^{(2)}(s+1) & =x^{(2)}(s)+x^{(1)}(s+1) \\
& =\sum_{i=1}^{s}(s+1-i) x^{(0)}(i)+\sum_{i=1}^{s+1} x^{(0)}(i) \\
& =\sum_{i=1}^{s+1}(s+1-i) x^{(0)}(i)+\sum_{i=1}^{s+1} x^{(0)}(i)
\end{aligned}
$$

$$
=\sum_{i=1}^{s+1}(s+2-i) x^{(0)}(i) . \text { It is also true. }
$$

So, Theorem 4 is true.

Theorem 5. Assume that the sequence of raw data is

$$
X^{(0)}=\left(x^{(0)}(1), x^{(0)}(2), \ldots, x^{(0)}(n)\right),
$$

where $r \in \mathbf{Z}^{+}$, and

$$
X^{(r)}=\left(x^{(r)}(1), x^{(r)}(2), \ldots, x^{(r)}(n)\right)
$$

is the rth integer order accumulating generation sequence of $X^{(0)}$, where

$$
\begin{array}{r}
x^{(r)}(k) \\
=\sum_{i=1}^{k} \frac{(k-i+1)(k-i+2) \cdots(k-i+r-1)}{(r-1) !} x^{(0)}(i), \\
k=1,2, \ldots, n .
\end{array}
$$

Proof. By induction on $r$, consider the following.

For $r=1, x^{(1)}(k)=\sum_{i=1}^{k}(((k-i+1)(k-i+2) \cdots(k-i+$ $r-1)) /(1-1) !) x^{(0)}(i)=\sum_{i=1}^{k} x^{(0)}(i)$. It is true.

For each $s \geq 1$, if $x^{(s)}(k)=\sum_{i=1}^{k}(((k-i+1)(k-i+2) \cdots(k-$ $i+s-1)) /(s-1) !) x^{(0)}(i)$ is true, it follows that

$$
\begin{aligned}
x^{(s+1)}(k)= & x^{(s+1)}(k-1)+x^{(s)}(k) \\
= & \sum_{i=1}^{k-1} \frac{(k-1-i+1)(k-1-i+2) \cdots(k-1-i+s+1-1)}{(s+1-1) !} x^{(0)}(i) \\
& +\sum_{i=1}^{k} \frac{(k-i+1)(k-i+2) \cdots(k-i+s-1)}{(s-1) !} x^{(0)}(i) \\
= & \sum_{i=1}^{k-1} \frac{(k-i)(k-i+1) \cdots(k-i+s-1)}{s !} x^{(0)}(i)+\sum_{i=1}^{k} \frac{(k-i+1)(k-i+2) \cdots(k-i+s-1)}{(s-1) !} x^{(0)}(i) \\
= & \sum_{i=1}^{k}\left(\frac{(k-i)(k-i+1) \cdots(k-i+s-1)}{s !}+\frac{(k-i+1)(k-i+2) \cdots(k-i+s-1)}{(s-1) !}\right) x^{(0)}(i) \\
= & \sum_{i=1}^{k} \frac{(k-i+1) \cdots(k-i+s-1)(k-i+s)}{s !} x^{(0)}(i) . \quad \text { It is also true. }
\end{aligned}
$$

So Theorem 5 is true for all $r \in \mathbf{Z}^{+}$.

Definition 6. For $n \in \mathbf{R}$ and $n \notin\{0,-1,-2,-3, \ldots\}$,

$$
\Gamma(n)=\int_{0}^{\infty} e^{-t} t^{n-1} d t
$$

is Gamma function for $n$.
Using integration by parts, the Gamma function satisfies the function

$$
\Gamma(n+1)=n \Gamma(n) .
$$

In particular, if $n$ is a positive integer,

$$
\Gamma(n+1)=\int_{0}^{\infty} e^{-t} t^{n} d t=n !
$$


For example, $\Gamma(1 / 2)=\sqrt{\pi}, \Gamma(1)=\Gamma(2)=1, \Gamma(3)=2$ ! , $\Gamma(4)=$ 3 !, $\ldots, \Gamma(n)=(n-1)$ !

The Gamma function is an extension of the factorial function.

By Gamma function, it follows that

$$
\begin{aligned}
x^{(r)} & (k) \\
& =\sum_{i=1}^{k} \frac{(k-i+1)(k-i+2) \cdots(k-i+r-1)}{(r-1) !} x^{(0)}(i) \\
& =\sum_{i=1}^{k} \frac{(r+k-i-1) !}{(k-i) !(r-1) !} x^{(0)}(i) \\
& =\sum_{i=1}^{k} \frac{\Gamma(r+k-i)}{\Gamma(k-i+1) \Gamma(r)} x^{(0)}(i) .
\end{aligned}
$$

Definition 7. Assume that the sequence of raw data is

$$
X^{(0)}=\left(x^{(0)}(1), x^{(0)}(2), \ldots, x^{(0)}(n)\right)
$$

where $r=\mathbf{R}^{+}$, and

$$
X^{(r)}=\left(x^{(r)}(1), x^{(r)}(2), \ldots, x^{(r)}(n)\right)
$$

is the $r$ th integer order accumulating generation sequence of $X^{(0)}$, where

$$
x^{(r)}(k)=\sum_{i=1}^{k} \frac{\Gamma(r+k-i)}{\Gamma(k-i+1) \Gamma(r)} x^{(0)}(i),
$$

$$
k=1,2, \ldots, n \text {. }
$$

Definition 8. Assume that

$$
X^{(0)}=\left(x^{(0)}(1), x^{(0)}(2), \ldots, x^{(0)}(n)\right)
$$

is a sequence of raw data and $D$ a sequence operator satisfying that

$$
X^{(0)} D=\left(x^{(0)}(1) d, x^{(0)}(2) d, \ldots, x^{(0)}(n) d\right),
$$

where

$$
x^{(0)}(k) d=x^{(0)}(k+1)-x^{(0)}(k), \quad k=1,2, \ldots, n,
$$

and the sequence operator $D$ is called the first-order forward reducing generation operator.

If

$$
x^{(0)}(k) d=x^{(0)}(k)-x^{(0)}(k-1), \quad k=1,2, \ldots, n,
$$

the sequence operator $D$ is called the first-order backward reducing generation operator.

By default, the reducing generation operator is a backward reducing generation operator. The first-order reducing generation operator is the first-order inverse accumulating generation operator (IAGO), written as 1-RGO.
Definition 9. For $r \in \mathbf{Z}^{+}$, we write

$$
X^{(-r)}=\left(x^{(-r)}(1), x^{(-r)}(2), \ldots, x^{(-r)}(n)\right)
$$

as the $r$ th-order reducing generation sequence of $X^{(0)}$, where

$$
\begin{aligned}
x^{(-r)}(k)=x^{(-r+1)}(k)-x^{(-r+1)}(k-1), & \\
& \quad k=1,2, \ldots, n .
\end{aligned}
$$

Definition 10. Assume that the sequence of raw data is

$$
\begin{aligned}
X^{(0)} & =\left(x^{(0)}(1), x^{(0)}(2), \ldots, x^{(0)}(n)\right), \\
X^{(-1)} & =\left(x^{(-1)}(1), x^{(-1)}(2), \ldots, x^{(-1)}(n)\right)
\end{aligned}
$$

and is the first-order reducing generation sequence of $X^{(0)}$, where

$$
x^{(-1)}(k)=x^{(0)}(k)-x^{(0)}(k-1), \quad k=1,2, \ldots, n,
$$

and then it follows that

$$
\begin{gathered}
X^{(-1)}=\left(x^{(0)}(1), x^{(0)}(2)-x^{(0)}(1), \ldots, x^{(0)}(k)\right. \\
\left.-x^{(0)}(k-1), \ldots, x^{(0)}(n)-x^{(0)}(n-1)\right) .
\end{gathered}
$$

From Definition 9, it obviously follows that

$$
x^{(-r-1)}(k)=x^{(-r)}(k)-x^{(-r)}(k-1),
$$

$$
k=1,2, \ldots, n \text {. }
$$

Then, it follows that

$$
\begin{aligned}
X^{(-2)} & =\left(x^{(-1)}(1), x^{(-1)}(2)-x^{(-1)}(1), \ldots, x^{(-1)}(k)\right. \\
& \left.-x^{(-1)}(k-1), \ldots, x^{(-1)}(n)-x^{(-1)}(n-1)\right) \\
& =\left(x^{(0)}(1),\left(x^{(0)}(2)-x^{(0)}(1)\right)-x^{(0)}(1), \ldots,\right. \\
& \left(x^{(0)}(k)-x^{(0)}(k-1)\right) \\
& -\left(x^{(0)}(k-1)-x^{(0)}(k-2)\right), \ldots, \\
& \left(x^{(0)}(n)-x^{(0)}(n-1)\right) \\
& \left.-\left(x^{(0)}(n-1)-x^{(0)}(n-2)\right)\right)=\left(x^{(0)}(1), x^{(0)}(2)\right. \\
& \left.-2 x^{(0)}(1)\right), \ldots, x^{(0)}(k)-2 x^{(0)}(k-1)+x^{(0)}(k \\
& -2), \ldots, x^{(0)}(n)-2 x^{(0)}(n-1)+x^{(0)}(n-2)
\end{aligned}
$$

So, $x^{(-2)}(k)=x^{(0)}(k)-2 x^{(0)}(k-1)+x^{(0)}(k-2)$,

$$
k=3,4, \ldots, n \text {. }
$$


Theorem 11. $X^{(-2)}=\left(x^{(-2)}(1), x^{(-2)}(2), \ldots, x^{(-2)}(n)\right)$ is the second-order reducing generation sequence of $X^{(0)}$, where

$$
\begin{aligned}
x^{(-2)}(k) & \begin{cases}\sum_{i=0}^{k-1}(-1)^{i} \frac{2}{(2-i) !} x^{(0)}(k-i) & k=1,2 \\
\sum_{i=0}^{2}(-1)^{i} \frac{2}{(2-i) !} x^{(0)}(k-i) & k=3,4, \ldots, n .\end{cases}
\end{aligned}
$$

Proof. By induction on $k$, consider the following.

For $k=1$,

$$
\begin{aligned}
x^{(-2)}(1) & =\sum_{i=0}^{k-1}(-1)^{i} \frac{2}{(2-i) !} x^{(0)}(k-i) \\
& =\sum_{i=0}^{0}(-1)^{i} \frac{2}{(2-i) !} x^{(0)}(1-i)=x^{(0)}(1) .
\end{aligned}
$$

It is true.

For $k=2$,

$$
\begin{aligned}
x^{(-2)}(2) & =\sum_{i=0}^{k-1}(-1)^{i} \frac{2}{(2-i) !} x^{(0)}(k-i) \\
& =\sum_{i=0}^{1}(-1)^{i} \frac{2}{(2-i) !} x^{(0)}(2-i) \\
& =x^{(0)}(2)-2 x^{(0)}(1) . \quad \text { It is true. }
\end{aligned}
$$

For $k=3,4, \ldots, n$,

$$
\begin{aligned}
x^{(-2)}(2) & =\sum_{i=0}^{r}(-1)^{i} \frac{r !}{i !(r-i) !} x^{(0)}(k-i) \\
& =\sum_{i=0}^{2}(-1)^{i} \frac{2}{i !(2-i) !} x^{(0)}(k-i) \\
& =x^{(0)}(k)-2 x^{(0)}(k-1)+x^{(0)}(k-2) .
\end{aligned}
$$

It is also true.

So, Theorem 11 is true.

Theorem 12. Assume that

$$
X^{(0)}=\left(x^{(0)}(1), x^{(0)}(2), \ldots, x^{(0)}(n)\right),
$$

where $r \in \mathbf{Z}^{+}$, and

$$
X^{(-r)}=\left(x^{(-r)}(1), x^{(-r)}(2), \ldots, x^{(-r)}(n)\right)
$$

is the rth integer order reducing generation sequence of $X^{(0)}$, where

$$
\begin{aligned}
& x^{(-r)}(k) \\
& = \begin{cases}\sum_{i=0}^{k-1}(-1)^{i} \frac{r !}{i !(r-i) !} x^{(0)}(k-i) & k=1,2, \ldots, r \\
\sum_{i=0}^{r}(-1)^{i} \frac{r !}{i !(r-i) !} x^{(0)}(k-i) & k=r+1, r+2, \ldots, n .\end{cases}
\end{aligned}
$$

Proof. By induction on $r$, consider the following. For $r=1$,

$$
\begin{aligned}
\begin{aligned}
\text { if } k & =1, \\
x^{(-1)}(1) & =\sum_{i=0}^{k-1}(-1)^{i} \frac{r !}{i !(r-i) !} x^{(0)}(k-i)=x^{(0)}(1) . \\
\text { If } k & =2,3, \ldots, n, \\
x^{(-1)}(k) & =\sum_{i=0}^{r}(-1)^{i} \frac{r !}{i !(r-i) !} x^{(0)}(k-i) \\
& =\sum_{i=0}^{1}(-1)^{i} \frac{1 !}{i !(1-i) !} x^{(0)}(k-i) \\
& =x^{(0)}(k)-x^{(0)}(k-1) . \quad \text { It is true. }
\end{aligned}
\end{aligned}
$$$$
\text { It is true; }
$$

For $r=2$,

$$
\begin{aligned}
\text { if } k & =1, \\
x^{(-2)}(1) & =\sum_{i=0}^{k-1}(-1)^{i} \frac{r !}{i !(r-i) !} x^{(0)}(k-i)=x^{(0)}(1) .
\end{aligned}
$$

It is true;

$$
\text { if } k=2 \text {, }
$$

$$
\begin{aligned}
x^{(-2)}(2) & =\sum_{i=0}^{k-1}(-1)^{i} \frac{r !}{i !(r-i) !} x^{(0)}(k-i) \\
& =\sum_{i=0}^{1}(-1)^{i} \frac{2 !}{i !(2-i) !} x^{(0)}(2-i) \\
& =x^{(0)}(2)-2 x^{(0)}(1) . \quad \text { It is true; }
\end{aligned}
$$

if $k=3,4, \ldots, n$,

$$
\begin{aligned}
x^{(-2)}(k) & =\sum_{i=0}^{r}(-1)^{i} \frac{r !}{i !(r-i) !} x^{(0)}(k-i) \\
& =\sum_{i=0}^{2}(-1)^{i} \frac{2 !}{i !(2-i) !} x^{(0)}(k-i) \\
& =x^{(0)}(k)-2 x^{(0)}(k-1)+x^{(0)}(k-2) .
\end{aligned}
$$

It is true.

For each $s \geq 1$, if

$$
\begin{aligned}
& x^{(-s)}(k) \\
& = \begin{cases}\sum_{i=0}^{k-1}(-1)^{i} \frac{s !}{i !(s-i) !} x^{(0)}(k-i) & k=1,2, \ldots, r \\
\sum_{i=0}^{s}(-1)^{i} \frac{s !}{i !(s-i) !} x^{(0)}(k-i) & k=s+1, s+2, \ldots, n\end{cases}
\end{aligned}
$$


is true, then it follows that

$$
\begin{aligned}
& x^{(-s-1)}(k)=x^{(-s)}(k)-x^{(-s)}(k-1) \\
& =\sum_{i=0}^{s}(-1)^{i} \frac{s !}{i !(s-i) !} x^{(0)}(k-i) \\
& -\sum_{i=0}^{s}(-1)^{i} \frac{s !}{i !(s-i) !} x^{(0)}(k-i-1)=\frac{s !}{s !} x^{(0)}(k) \\
& -\frac{s !}{1 !(s-1) !} x^{(0)}(k-1)+\frac{s !}{2 !(s-2) !} x^{(0)}(k-2) \\
& +(-1)^{i} \frac{s !}{i !(s-i) !} x^{(0)}(k-i)+(-1)^{s} \frac{s !}{s !(s-s) !} \\
& \cdot x^{(0)}(k-s)-\frac{s !}{s !} x^{(0)}(k-1)+\frac{s !}{1 !(s-1) !} \\
& \cdot x^{(0)}(k-2)-\frac{s !}{2 !(s-2) !} x^{(0)}(k-3)-(-1)^{i+1} \\
& \frac{s !}{(i+1) !(s-i-1) !} x^{(0)}(k-i)-(-1)^{s} \frac{s !}{s !(s-s) !} \\
& \cdot x^{(0)}(k-s-1)=\frac{s !}{s !} x^{(0)}(k)-\left(\frac{s !}{1 !(s-1) !}+\frac{s !}{s !}\right) \\
& \cdot x^{(0)}(k-1)+\left(\frac{s !}{2 !(s-2) !}+\frac{s !}{1 !(s-1) !}\right) \\
& \cdot x^{(0)}(k-2) \\
& +\left((-1)^{i} \frac{s !}{i !(s-i) !}+(-1)^{i+1} \frac{s !}{(i-1) !(s-i+1) !}\right) \\
& \cdot x^{(0)}(k-i)-(-1)^{s} \frac{s !}{s !(s-s) !} x^{(0)}(k-s-1) \text {. }
\end{aligned}
$$

Then, it follows that

$$
\begin{aligned}
x^{(-s-1)}(k)= & x^{(-s)}(k)-x^{(-s)}(k-1) \\
= & \sum_{i=0}^{s}(-1)^{i} \frac{s !}{i !(s-i) !} x^{(0)}(k-i) \\
& -\sum_{i=0}^{s}(-1)^{i} \frac{s !}{i !(s-i) !} x^{(0)}(k-i-1) .
\end{aligned}
$$

The coefficient of $x^{(0)}(k-i)$ for the expansion of $x^{(-s-1)}(k)$ is

$$
\begin{aligned}
(-1)^{i} \frac{s !}{i !(s-i) !}-(-1)^{i-1} \frac{s !}{(i-1) !(s-i+1) !}=(-1)^{i} \\
\cdot \frac{s !}{i !(s-i) !}+(-1)^{i} \frac{s !}{(i-1) !(s-i+1) !}=(-1)^{i} \\
\quad \cdot\left(\frac{s !}{i !(s-i) !}+\frac{s !}{(i-1) !(s-i+1) !}\right)=(-1)^{i} \\
\cdot\left(\frac{s !(s-i+1)}{i !(s-i) !(s-i+1)}+\frac{i \cdot s !}{i \cdot(i-1) !(s-i+1) !}\right)
\end{aligned}
$$

$$
\begin{aligned}
& =(-1)^{i} \frac{s !(s-i+1+i)}{i !(s-i+1) !}=(-1)^{i} \frac{s !(s+1)}{i !(s-i+1) !} \\
& =(-1)^{i} \frac{(s+1) !}{i !(s-i+1) !} .
\end{aligned}
$$

So, $x^{(-s-1)}(k)=\sum_{i=0}^{s}(-1)^{i}((s+1) ! / i !(s-i+1) !) x^{(0)}(k-i)$. So, Theorem 12 is true.

Proposition 13. For $r \in \mathbf{Z}^{+}$, the rth integer order reducing generation operator

$$
\begin{aligned}
& x^{(-r)}(k) \\
& = \begin{cases}\sum_{i=0}^{k-1}(-1)^{i} \frac{r !}{i !(r-i) !} x^{(0)}(k-i) & k=1,2, \ldots, r \\
\sum_{i=0}^{r}(-1)^{i} \frac{r !}{i !(r-i) !} x^{(0)}(k-i) & k=r+1, r+2, \ldots, n\end{cases}
\end{aligned}
$$

can be simplified as

$$
\begin{array}{r}
x^{(-r)}(k)=\sum_{i=0}^{k-1}(-1)^{i} \frac{\Gamma(r+1)}{\Gamma(i+1) \Gamma(r-i+1)} x^{(0)}(k-i), \\
k=1,2, \ldots, n .
\end{array}
$$

Proof. According to the property of Gamma function, $\Gamma(n+$ 1) $=n$ !

When $1 \leq k \leq r$,

$$
\begin{aligned}
x^{(-r)}(k) & =\sum_{i=0}^{k-1}(-1)^{i} \frac{r !}{i !(r-i) !} x^{(0)}(k-i) \\
& =\sum_{i=0}^{k-1}(-1)^{i} \frac{\Gamma(r+1)}{\Gamma(i+1) \Gamma(r-i+1)} x^{(0)}(k-i) .
\end{aligned}
$$

It is true.

When $r+1 \leq k \leq n$,

$$
\begin{aligned}
& \text { for } k-i \geq 1 \text {, then } i \leq k-1 ; \\
& \text { for } r+1 \leq i \leq k-1, \text { then } r ! / i !(r-i) !=0 .
\end{aligned}
$$

Thus, it follows that

$$
\begin{aligned}
x^{(-r)}(k)= & \sum_{i=0}^{r}(-1)^{i} \frac{r !}{i !(r-i) !} x^{(0)}(k-i) \\
= & \sum_{i=0}^{r}(-1)^{i} \frac{r !}{i !(r-i) !} x^{(0)}(k-i) \\
& +\sum_{i=r+1}^{k-1}(-1)^{i} \frac{r !}{i !(r-i) !} x^{(0)}(k-i) \\
= & \sum_{i=0}^{k-1}(-1)^{i} \frac{\Gamma(r+1)}{\Gamma(i+1) \Gamma(r-i+1)} x^{(0)}(k-i) .
\end{aligned}
$$

It is also true.

So, Proposition 13 is true. 
Definition 14. Assume that

$$
X^{(0)}=\left(x^{(0)}(1), x^{(0)}(2), \ldots, x^{(0)}(n)\right),
$$

where $r \in \mathbf{R}^{+}$, and

$$
X^{(-r)}=\left(x^{(-r)}(1), x^{(-r)}(2), \ldots, x^{(-r)}(n)\right)
$$

is the $r$ th integer order reducing generation sequence of $X^{(0)}$, where

$$
\begin{array}{r}
x^{(-r)}(k)=\sum_{i=0}^{k-1}(-1)^{i} \frac{\Gamma(r+1)}{\Gamma(i+1) \Gamma(r-i+1)} x^{(0)}(k-i), \\
k=1,2, \ldots, n .
\end{array}
$$

Theorem 15. Assume that

$$
X^{(0)}=\left(x^{(0)}(1), x^{(0)}(2), \ldots, x^{(0)}(n)\right)
$$

is a sequence of raw data, where $p \in \mathbf{R}^{+}$and $q \in \mathbf{R}^{+} . X^{(p)}$ is the sequence of the pth-order accumulating generation of $X^{(0)} . X^{(-q)}$ is the sequence of the qth-order reducing generation of $X^{(0)} .\left(X^{(p)}\right)^{(-q)}$ is the sequence of the qth-order reducing generation of $X^{(p)} .\left(X^{(-q)}\right)^{(p)}$ is the sequence of the pth-order accumulating generation of $X^{(-q)}$. The following holds true [19]:

(1) If $p-q>0, X^{(p-q)}$ is the $p-q$ order accumulating generation of $X^{(0)}$.

(2) If $p-q<0, X^{(p-q)}$ is the $q-p$ order accumulating generation of $X^{(0)}$.

(3) The fractional accumulating generation operator and the fractional reducing generation operator satisfy the exchange law and the exponential rate.

$$
X^{(p-q)}=\left(X^{(p)}\right)^{(-q)}=\left(X^{(-q)}\right)^{(p)} .
$$

Theorem 16. Assume that

$$
X^{(0)}=\left(x^{(0)}(1), x^{(0)}(2), \ldots, x^{(0)}(n)\right)
$$

is a sequence of raw data and $r \in \mathbf{R}^{+} . X^{(r)}$ is the sequence of the rth-order accumulating generation of $X^{(0)} . X^{(-r)}$ is the sequence of the rth-order reducing generation of $X^{(0)}$. The rth order accumulating generation operator and the rth-order reducing generation operator are inverse operations [19].

$$
X^{(0)}=\left(X^{(r)}\right)^{(-r)}=\left(X^{(-r)}\right)^{(r)} .
$$

\section{Discrete Grey Model with Fractional Operators}

Definition 17. Assume that $X^{(0)}$ and $X^{(r)}$ are defined as in Definition 7, and

$$
x^{(r)}(k+1)=\beta_{1} x^{(r)}(k)+\beta_{2}
$$

is the discrete grey model with fractional operators. In particular, consider the following.

$$
\begin{gathered}
\text { If } r=1, x^{(r)}(k+1)=\beta_{1} x^{(r)}(k)+\beta_{2} \text { is } \operatorname{DGM}(1,1) . \\
x^{(1)}(k+1)=\beta_{1} x^{(1)}(k)+\beta_{2} .
\end{gathered}
$$

If $r=0, x^{(r)}(k+1)=\beta_{1} x^{(r)}(k)+\beta_{2}$ is direct modeling $\operatorname{DGM}(1,1)$.

$$
x^{(0)}(k+1)=\beta_{1} x^{(0)}(k)+\beta_{2} .
$$

Theorem 18. Assume that

$$
X^{(0)}=\left(x^{(0)}(1), x^{(0)}(2), \ldots, x^{(0)}(n)\right)
$$

is a sequence of raw data and

$$
x^{(r)}(k)=\sum_{i=1}^{k} \frac{\Gamma(r+k-i)}{\Gamma(k-i+1) \Gamma(r)} x^{(0)}(i),
$$

$$
k=1,2, \ldots, n
$$

is the sequence of the rth-order accumulating generation of $X^{(0)}$, where $r \in \mathbf{R}^{+}$.

$$
X^{(r)}=\left(x^{(r)}(1), x^{(r)}(2), \ldots, x^{(r)}(n)\right)
$$

is the rth-order accumulating generation of $X^{(0)}$, where

$$
x^{(r)}(k)=\sum_{i=1}^{k} \frac{\Gamma(r+k-i)}{\Gamma(k-i+1) \Gamma(r)} x^{(0)}(i),
$$

$$
k=1,2, \ldots, n \text {. }
$$

The parameter vector $\widehat{\boldsymbol{\beta}}=\left[\beta_{1}, \beta_{2}\right]^{\mathrm{T}}$ of discrete grey model with fractional operators $x^{(r)}(k+1)=\beta_{1} x^{(r)}(k)+\beta_{2}$ can be calculated by the least squares method.

$$
\widehat{\boldsymbol{\beta}}=\left(\mathbf{B}^{\mathrm{T}} \mathbf{B}\right)^{-1} \mathbf{B}^{\mathrm{T}} \mathbf{Y}
$$

It follows that

$$
\mathbf{Y}=\left[\begin{array}{c}
x^{(r)}(2) \\
x^{(r)}(3) \\
\vdots \\
x^{(r)}(n)
\end{array}\right]
$$

$$
\mathbf{B}=\left[\begin{array}{cc}
x^{(r)}(1) & 1 \\
x^{(r)}(2) & 1 \\
\vdots & \vdots \\
x^{(r)}(n-1) & 1
\end{array}\right] \text {, }
$$

where

$$
x^{(r)}(k)=\sum_{i=1}^{k} \frac{\Gamma(r+k-i)}{\Gamma(k-i+1) \Gamma(r)} x^{(0)}(i),
$$

$$
k=1,2, \ldots, n \text {. }
$$


So, it follows that

$$
\begin{aligned}
& \mathbf{Y}=\left[\begin{array}{c}
x^{(r)}(2) \\
x^{(r)}(3) \\
\vdots \\
x^{(r)}(n)
\end{array}\right]=\left[\begin{array}{c}
\sum_{i=1}^{2} \frac{\Gamma(r+2-i)}{\Gamma(2-i+1) \Gamma(r)} x^{(0)}(i) \\
\sum_{i=1}^{3} \frac{\Gamma(r+3-i)}{\Gamma(3-i+1) \Gamma(r)} x^{(0)}(i) \\
\vdots \\
\sum_{i=1}^{n} \frac{\Gamma(r+n-i)}{\Gamma(n-i+1) \Gamma(r)} x^{(0)}(i)
\end{array}\right] \\
& =\left[\begin{array}{c}
r x^{(0)}(1)+x^{(0)}(2) \\
\frac{r(r+1)}{2} x^{(0)}(1)+r x^{(0)}(2)+x^{(0)}(3) \\
\vdots \\
\sum_{i=1}^{n} \frac{\Gamma(r+n-i)}{\Gamma(n-i+1) \Gamma(r)} x^{(0)}(i)
\end{array}\right] .
\end{aligned}
$$$$
\mathbf{B}=\left[\begin{array}{cc}
x^{(r)}(1) & 1 \\
x^{(r)}(2) & 1 \\
\vdots & \vdots \\
x^{(r)}(n-1) & 1
\end{array}\right]
$$$$
=\left[\begin{array}{cc}
\sum_{i=1}^{1} \frac{\Gamma(r+1-i)}{\Gamma(1-i+1) \Gamma(r)} x^{(0)}(i) & 1 \\
\sum_{i=1}^{2} \frac{\Gamma(r+2-i)}{\Gamma(2-i+1) \Gamma(r)} x^{(0)}(i) & 1 \\
\vdots & \vdots \\
\sum_{i=1}^{n-1} \frac{\Gamma(r+n-1-i)}{\Gamma(n-1-i+1) \Gamma(r)} x^{(0)}(i) & 1
\end{array}\right]
$$$$
=\left[\begin{array}{cc}
x^{(0)}(1) & 1 \\
r x^{(0)}(1)+x^{(0)}(2) & 1 \\
\vdots & \vdots \\
\sum_{i=1}^{n-1} \frac{\Gamma(r+n-1-i)}{\Gamma(n-1-i+1) \Gamma(r)} x^{(0)}(i) & 1
\end{array}\right]
$$

Theorem 19. Assume that $\mathbf{B}, \mathbf{Y}$, and $\widehat{\boldsymbol{\beta}}$ are the same as in Theorem 18. If

$$
\widehat{\boldsymbol{\beta}}=\left[\beta_{1}, \beta_{2}\right]^{\mathrm{T}}=\left(\mathbf{B}^{\mathrm{T}} \mathbf{B}\right)^{-1} \mathbf{B}^{\mathrm{T}} \mathbf{Y},
$$

then the following is true.

(1) The time response sequence of the discrete grey model with fractional operators

$$
x^{(r)}(k+1)=\beta_{1} x^{(r)}(k)+\beta_{2}
$$

is given by

$$
\begin{aligned}
\widehat{x}^{(r)}(k)=\left(x^{(0)}(1)-\frac{\beta_{2}}{1-\beta_{1}}\right) \beta_{1}{ }^{k-1}+ & \frac{\beta_{2}}{1-\beta_{1}}, \\
& k=2,3, \ldots, n
\end{aligned}
$$

or

$$
\begin{aligned}
\hat{x}^{(r)}(k)=x^{(0)}(1) \beta_{1}{ }^{k-1}+\frac{1-\beta_{1}{ }^{k-1}}{1-\beta_{1}} \beta_{2}, & \\
& k=2,3, \ldots, n .
\end{aligned}
$$

(2) The restored values of $x^{(0)}(k)$ can be given by

$$
\begin{array}{rl}
\widehat{x}^{(0)}(k) & =\left(\widehat{x}^{(r)}\right)^{(-r)}(k) \\
& =\sum_{i=0}^{k-1}(-1)^{i} \frac{\Gamma(r+1)}{\Gamma(i+1) \Gamma(r-i+1)} \widehat{x}^{(r)}(k-i), \\
k & k=2,3, \ldots, n \\
\widehat{x}^{(0)}(1)= & x^{(0)}(1) .
\end{array}
$$

Theorem 20. Discrete grey model $\operatorname{DGM}(1,1)$ is the special case of the discrete grey model with fractional operators, where $r=1$.

The conclusion is clearly true.

To determine the optimal order of the discrete grey model with fractional operators, we can solve the following optimization problem:

$$
\min f(r)=\frac{1}{n-1} \sum_{k=2}^{n} \frac{\left|x^{(0)}(k)-\widehat{x}^{(0)}(k)\right|}{x^{(0)}(k)}, \quad r \in \mathbf{R}^{+} .
$$

Genetic algorithms have obvious advantages at solving nonlinear optimization problems [30]. So, we can apply genetic algorithms to solve the optimized value of order $r$.

Step 1 (start). Initialize the parameters of population $(t)$ : initial value of order $r$, range of value for $r$, population size $N$, crossover probability pc, mutation probability pm, value accuracy represented as ee, fitness function results accuracy re, and generations of terminating evolution $T$.

Step 2 (fitness). Determine the fitness of population $(t)$.

Step 2.1. Compute the $r$ order accumulating generation sequence $X^{(r)}$.

Step 2.2. Compute the parameter vector $\widehat{\boldsymbol{\beta}}$.

Step 2.3. Compute the time response sequence $\widehat{x}^{(r)}(k)$.

Step 2.4. Compute the forecast value $\widehat{x}^{(0)}(k)$.

Step 2.5. Compute the fitness $f(r)$. 


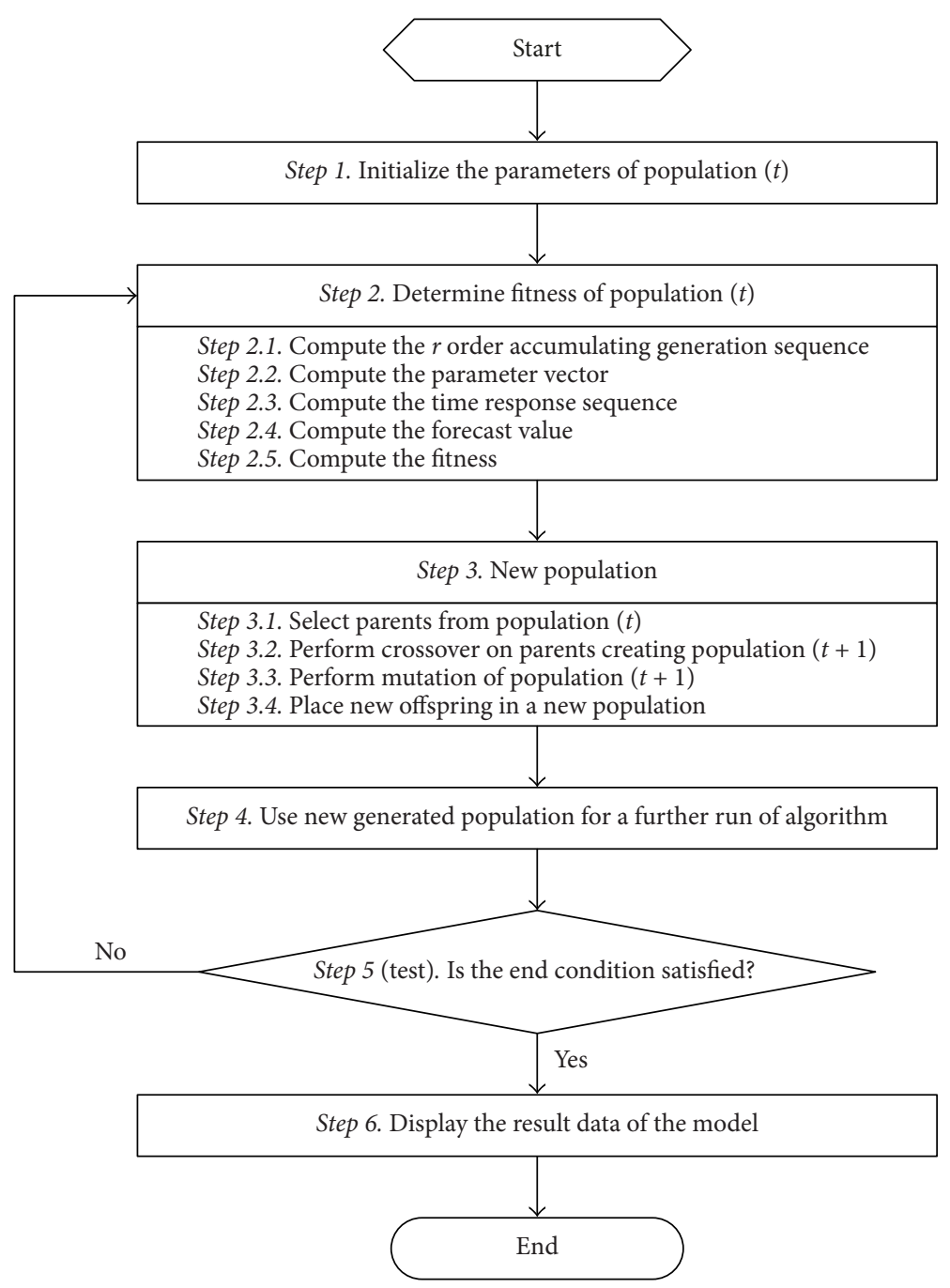

FIGURE 1: The modeling steps of discrete grey model with optimized fractional operators.

Step 3. New population.

Step 3.1 (selection). Select parents from population $(t)$.

Step 3.2 (crossover). Perform crossover on parents creating population $(t+1)$.

Step 3.3 (mutation). Perform mutation of population $(t+1)$.

Step 3.4 (accepting). Place new offspring in a new population.

Step 4 (replace). Use new generated population for a further run of algorithm.

Step 5 (test). If the end condition is satisfied, stop, and return the best solution in current population; otherwise go to Step 2.

Step 6 (result). Display the result data of the model, the optimized order $r$, the parameter vector $\widehat{\boldsymbol{\beta}}$, and the forecast value $\widehat{x}^{(0)}(k)$.
The overall modeling steps of the discrete grey model with optimized fractional operators are shown in Figure 1.

\section{Verification of Discrete Grey Model with Fractional Operators}

In this section, the advantage of the discrete grey model with fractional operators over the other discrete grey models is demonstrated by three case studies. Mean absolute percentage error (MAPE) is used to compare the actual values with forecasted values to evaluate the precision. MAPE is defined as

$$
\mathrm{MAPE}=\frac{1}{n} \sum_{k=1}^{n}\left|\frac{x(k)-\widehat{x}(k)}{x(k)}\right| \times 100 \%
$$

Case 1. In [31], a modified $\operatorname{DGM}(1,1)$ was proposed to predict China's foreign exchange reserves. We consider the example from this paper [31] which provides the sample data. The actual values and the forecast values of the three 
TABLE 1: The fitted values and MAPE of different discrete grey models (unit: \$ billion).

\begin{tabular}{|c|c|c|c|c|c|c|c|}
\hline \multirow{2}{*}{ Year } & \multirow{2}{*}{ Actual value $x^{(0)}(k)$} & \multicolumn{2}{|c|}{$\operatorname{DGM}(1,1)$} & \multicolumn{2}{|c|}{ Modified DGM $(1,1)$} & \multicolumn{2}{|c|}{$r=0.020$} \\
\hline & & $\widehat{x}^{(0)}(k)$ & $\Delta_{k}(\%)$ & $\widehat{x}^{(0)}(k)$ & $\Delta_{k}(\%)$ & $\widehat{x}^{(0)}(k)$ & $\Delta_{k}(\%)$ \\
\hline 1994 & 51.620 & & & & & & \\
\hline 1995 & 73.597 & 25.687 & 65.098 & 94.227 & 28.031 & 82.846 & 12.567 \\
\hline 1996 & 105.049 & 34.109 & 67.530 & 106.679 & 1.552 & 107.697 & 2.521 \\
\hline 1997 & 139.890 & 45.294 & 67.622 & 120.778 & 13.662 & 127.487 & 8.866 \\
\hline 1998 & 144.959 & 60.145 & 58.509 & 136.740 & 5.670 & 143.251 & 1.178 \\
\hline 1999 & 154.675 & 79.867 & 48.365 & 154.811 & 0.088 & 155.805 & 0.731 \\
\hline \multirow[t]{2}{*}{2000} & 165.574 & 106.055 & 35.947 & 175.271 & 5.856 & 165.796 & 0.134 \\
\hline & \multicolumn{3}{|l|}{ MAPE (\%) } & \multicolumn{2}{|c|}{9.143} & \multicolumn{2}{|c|}{4.333} \\
\hline 2001 & 212.165 & 140.831 & 33.622 & & & \multicolumn{2}{|c|}{$r=0.662$} \\
\hline 2002 & 286.407 & 187.009 & 34.705 & 288.721 & 0.808 & 303.083 & 5.823 \\
\hline 2003 & 403.251 & 248.329 & 38.418 & 407.365 & 1.020 & 431.186 & 6.927 \\
\hline 2004 & 609.932 & 329.756 & 45.936 & 574.762 & 5.766 & 594.877 & 2.468 \\
\hline 2005 & 818.872 & 437.882 & 46.526 & 810.949 & 0.968 & 806.887 & 1.464 \\
\hline \multirow[t]{3}{*}{2006} & 1066.300 & 581.463 & 45.469 & 1144.191 & 7.305 & 1083.648 & 1.627 \\
\hline & & \multicolumn{2}{|c|}{ MAPE (\%) } & \multicolumn{2}{|c|}{4.846} & \multicolumn{2}{|c|}{3.662} \\
\hline & MAPE (\%) & \multicolumn{2}{|c|}{48.979} & \multicolumn{2}{|c|}{7.190} & \multicolumn{2}{|c|}{4.028} \\
\hline
\end{tabular}

TABLE 2: The fitted values and MAPE of different discrete grey models.

\begin{tabular}{|c|c|c|c|c|}
\hline Sequence & Sample data & Geometric shape of sequence & Models & MAPE (\%) \\
\hline \multirow{3}{*}{$X_{1}$} & \multirow{3}{*}{$(1.2,2.9,4.2,5.1,5.8)$} & \multirow{3}{*}{ Up, convex } & Direct modeling DGM $(1,1)$ & 0.2958 \\
\hline & & & $r=1$ & 6.1492 \\
\hline & & & $r=0$ & 0.29549 \\
\hline \multirow{3}{*}{$X_{2}$} & \multirow{3}{*}{$(8.5,16.4,32.3,64.2,128.1)$} & \multirow{3}{*}{ Up, concave } & Direct modeling DGM $(1,1)$ & 0.1754 \\
\hline & & & $r=1$ & 0.5621 \\
\hline & & & $r=0.018$ & 0.0086 \\
\hline \multirow{3}{*}{$X_{3}$} & \multirow{3}{*}{$(5.8,5.1,4.2,2.9,1.2)$} & \multirow{3}{*}{ Down, convex } & Direct modeling DGM $(1,1)$ & 0.3321 \\
\hline & & & $r=1$ & 19.1751 \\
\hline & & & $r=0$ & 0.33136 \\
\hline \multirow{3}{*}{$X_{4}$} & \multirow{3}{*}{$(128.1,64.2,32.3,16.4,8.5)$} & \multirow{3}{*}{ Down, concave } & Direct modeling DGM $(1,1)$ & 0.0756 \\
\hline & & & $r=1$ & 0.7371 \\
\hline & & & $r=0.985$ & 0.0258 \\
\hline \multirow{3}{*}{$X_{5}$} & \multirow{3}{*}{$(5,11,29,83,245)$} & \multirow{3}{*}{ Strict nonhomogeneous } & Direct modeling DGM $(1,1)$ & 0.0000 \\
\hline & & & $r=1$ & 13.8393 \\
\hline & & & $r=0$ & 0.0000 \\
\hline \multirow{3}{*}{$X_{6}$} & \multirow{3}{*}{$(1.4,2,2.8,3.9,5.4)$} & \multirow{3}{*}{ Approximate nonhomogeneous } & Direct modeling DGM $(1,1)$ & 0.1099 \\
\hline & & & $r=1$ & 0.2766 \\
\hline & & & $r=0.943$ & 0.0467 \\
\hline
\end{tabular}

compared models are presented in Table 1. As can be seen from Table 1 , the discrete grey model with fractional operators where $r=0.020$ and $r=0.662$ achieved the lowest MAPE compared to $\operatorname{DGM}(1,1)$ and the modified $\operatorname{DGM}(1,1)$.

Case 2. In [32], the initial condition of $\operatorname{DGM}(1,1)$ was optimized and the results of direct modeling $\operatorname{DGM}(1,1)$ were tested against the original data, in respect of a geometric shape with up, down, concave, and convex sequences. Sample data from [32] were used in this case study. The actual values and the forecast values of three compared models are presented in Table 2. Again, the discrete grey model with fractional operators achieved the lowest MAPE compared to $\operatorname{DGM}(1,1)$ (where $r=1$ ) and direct modeling $\operatorname{DGM}(1,1)$.

Case 3. The authors of [25] proposed a discrete grey model based on fractional order accumulation and studied the turnover of goods in Jiangsu Province, China. We consider 
TABLE 3: The actual values of the turnover of goods in Jiangsu Province, China.

\begin{tabular}{lccccccc}
\hline Year & 2003 & 2004 & 2005 & 2006 & 2007 & 2008 & 2009 \\
\hline Actual value (unit: billion tons-km) & 181.74 & 239.81 & 306.83 & 364.41 & 409.84 & 470.75 & 515.45 \\
\hline
\end{tabular}

TABLE 4: The forecast values and errors of different discrete grey models (year 2009).

\begin{tabular}{|c|c|c|c|c|}
\hline Model & $\operatorname{DGM}^{1 / 2}(1,1)$ & $\operatorname{DGM}^{2 / 3}(1,1)$ & $\operatorname{DGM}(1,1)$ & $r=0.27$ \\
\hline Forecast value (unit: billion tons-km) & 5236.45 & 5303.33 & 5585.60 & 5185.92 \\
\hline Absolute percentage error (\%) & 1.59 & 2.89 & 8.36 & 0.61 \\
\hline
\end{tabular}

TABLE 5: Fitting accuracy of China's sulfur dioxide emissions (unit: million tons).

\begin{tabular}{|c|c|c|c|c|c|c|c|c|c|c|}
\hline \multirow{3}{*}{$k$} & \multirow{3}{*}{ Year } & \multirow{3}{*}{$x^{(0)}(k)$} & \multirow{2}{*}{\multicolumn{2}{|c|}{$\operatorname{GM}(1,1)$}} & \multicolumn{6}{|c|}{ Fractional order $\operatorname{DGM}(1,1)$} \\
\hline & & & & & \multicolumn{2}{|c|}{$r=1$} & \multicolumn{2}{|c|}{$r=0$} & \multicolumn{2}{|c|}{$r=0.07$} \\
\hline & & & $\hat{x}^{(0)}(k)$ & $\Delta_{k}(\%)$ & $\widehat{x}^{(0)}(k)$ & $\Delta_{k}(\%)$ & $\hat{x}^{(0)}(k)$ & $\Delta_{k}(\%)$ & $\hat{x}^{(0)}(k)$ & $\Delta_{k}(\%)$ \\
\hline 1 & 2007 & 24.681 & & & & & & & & \\
\hline 2 & 2008 & 23.212 & 23.021 & 0.824 & 23.024 & 0.811 & 23.246 & 0.147 & 23.21 & 0.007 \\
\hline 3 & 2009 & 22.144 & 22.532 & 1.752 & 22.534 & 1.762 & 22.299 & 0.701 & 22.387 & 1.097 \\
\hline 4 & 2010 & 21.851 & 22.054 & 0.927 & 22.055 & 0.933 & 21.674 & 0.808 & 21.862 & 0.050 \\
\hline 5 & 2011 & 22.179 & 21.585 & 2.676 & 21.586 & 2.675 & 21.262 & 4.134 & 21.478 & 3.161 \\
\hline 6 & 2012 & 21.176 & 21.127 & 0.231 & 21.127 & 0.233 & 20.99 & 0.878 & 21.177 & 0.003 \\
\hline 7 & 2013 & 20.440 & 20.679 & 1.167 & 20.677 & 1.161 & 20.811 & 1.813 & 20.929 & 2.392 \\
\hline \multicolumn{3}{|c|}{ Average relative error $\Delta$} & \multicolumn{2}{|c|}{$1.263 \%$} & \multicolumn{2}{|c|}{$1.262 \%$} & \multicolumn{2}{|c|}{$1.413 \%$} & \multicolumn{2}{|c|}{$1.118 \%$} \\
\hline
\end{tabular}

TABLE 6: Model testing result of China's sulfur dioxide emissions (unit: million tons).

\begin{tabular}{|c|c|c|c|c|c|c|c|c|c|c|}
\hline \multirow{3}{*}{$k$} & \multirow{3}{*}{ Year } & \multirow{3}{*}{$x^{(0)}(k)$} & \multirow{2}{*}{\multicolumn{2}{|c|}{$\mathrm{GM}(1,1)$}} & \multicolumn{6}{|c|}{ Fractional Order DGM $(1,1)$} \\
\hline & & & & & \multicolumn{2}{|c|}{$r=1$} & \multicolumn{2}{|c|}{$r=0$} & \multicolumn{2}{|c|}{$r=0.07$} \\
\hline & & & $\widehat{x}^{(0)}(k)$ & $\Delta_{k}(\%)$ & $\widehat{x}^{(0)}(k)$ & $\Delta_{k}(\%)$ & $\widehat{x}^{(0)}(k)$ & $\Delta_{k}(\%)$ & $\widehat{x}^{(0)}(k)$ & $\Delta_{k}(\%)$ \\
\hline 8 & 2014 & 19.744 & 20.240 & 2.512 & 20.237 & 2.497 & 20.369 & 3.166 & 19.642 & 0.517 \\
\hline 9 & 2015 & 18.591 & 19.413 & 4.405 & 19.807 & 6.541 & 19.686 & 5.890 & 18.848 & 1.382 \\
\hline
\end{tabular}

the sample data from this paper as an example. The actual values and the forecast values of the different DGM models being compared are presented in Tables 3 and 4 . The results show that the discrete grey model with fractional operators has a lower MAPE than the discrete grey model based on fractional order accumulate.

\section{Prediction of China's Sulfur Dioxide Emissions}

According to China's environmental status report published in 2016, from 2007 to 2015, the sulfur dioxide emission data (unit, million tons) are as follows:

$$
X^{(0)}=(24.681,23.212,22.144,21.851,22.179,21.176,20.440,19.744,18.591)
$$

The order of discrete grey model with fractional order operators can be resolved by the algorithm of particle swarm optimization. The minimum mean relative error is thus obtained. The results of discrete grey model with fractional order operators where $r=0.07, \operatorname{DGM}(1,1)$ model (discrete grey model with fractional order operators where $r=$ 1 ), direct modeling $\operatorname{DGM}(1,1)$ (discrete grey model with fractional order operators where $r=0)$, and $\operatorname{GM}(1,1)$ are shown in Table 5. From Tables 5 and 6, we can see that the discrete grey model with fractional order operators where $r=0.07$ achieves the best fitting accuracy and test accuracy among the four models. The average relative error of discrete grey model with fractional order operators where $r=0.07$ is $1.118 \%$. By using discrete grey model with fractional order operators where $r=0.07$, the predicted values of sulfur dioxide emissions in China in the next five years are shown in Table 7.

According to the predicted results of China's sulfur dioxide emissions shown in Table 7 , the emissions show a steady decline in the five years from 2016 to 2020. By 2020, the amount of sulfur dioxide emissions is 16.145 million tons, only $86.843 \%$ of emissions in 2015 . The result indicates that China's 
TABle 7: Prediction of China's sulfur dioxide emissions (unit: million tons).

\begin{tabular}{lccccc}
\hline Year & 2016 & 2017 & 2018 & 2019 & 2020 \\
\hline Forecast value & 17.926 & 17.637 & 17.145 & 16.569 & 16.145 \\
\hline
\end{tabular}

current sulfur dioxide emission reduction policies have been well implemented and achieved a positive outcome.

\section{Conclusion}

In this paper, the discrete grey model with fractional operators is firstly studied and then used to predict China's sulfur dioxide emissions. This paper introduces a new prediction model by changing and optimizing the values of fractional order, giving rise to a discrete grey model with fractional operators. The steps of modeling and genetic algorithms of order optimization for the proposed model are explained. Case studies show that the discrete grey model with fractional operators can achieve the best precision with optimized order. Then, China's future sulfur dioxide emissions are predicted by the discrete grey model with fractional operators. Genetic algorithm can be used to determine the optimal order under the condition of minimum mean error. The results show that the proposed discrete grey model with fractional operators can obtain a higher fitting accuracy than the grey model $\operatorname{GM}(1,1)$, discrete grey model $\operatorname{DGM}(1,1)$, and direct modeling $\operatorname{DGM}(1,1)$. The forecast results show that China's sulfur dioxide emissions will decrease steadily, indicating that China's sulfur dioxide reduction policies are effective. It is noted that the fractional order accumulating generation operator and fractional order reducing generation operator may have the same expression. The unified expression of fractional order accumulating/reducing generation operators is an attractive topic in the grey system theory.

\section{Conflicts of Interest}

The authors declare that there are no conflicts of interest regarding the publication of this paper.

\section{Acknowledgments}

This work is supported by the National Natural Science Foundation of China (71771033), Humanity and Social Science Foundation of the Ministry of Education of China (14YJAZH033), Project Foundation of Chongqing Municipal Education Committee (KJ1706166), and Research Fund of Chongqing Technology and Business University (670101485, KFJJ2017059).

\section{References}

[1] F. García-Labiano, L. F. de Diego, A. Cabello et al., "Sulphuric acid production via Chemical Looping Combustion of elemental sulphur," Applied Energy, vol. 178, pp. 736-745, 2016.

[2] K. A. Evans, P. K. Hopke, M. J. Utell et al., "Triggering of STelevation myocardial infarction by ambient wood smoke and other particulate and gaseous pollutants," Journal of Exposure Science and Environmental Epidemiology, vol. 27, no. 2, pp. 198206, 2017.

[3] N. Sheng and U. W. Tang, "The first official city ranking by air quality in China-a review and analysis," Cities, vol. 51, pp. 139149, 2016.

[4] Z. Lu, D. G. Streets, Q. Zhang et al., "Sulfur dioxide emissions in China and sulfur trends in East Asia since 2000," Atmospheric Chemistry and Physics, vol. 10, no. 13, pp. 6311-6331, 2010.

[5] Z. Lu, Q. Zhang, and D. G. Streets, "Sulfur dioxide and primary carbonaceous aerosol emissions in China and India, 1996-2010," Atmospheric Chemistry and Physics, vol. 11, no. 18, pp. 98399864, 2011.

[6] S. J. Smith, H. Pitchera, and T. M. L. Wigley, "Global and regional anthropogenic sulfur dioxide emissions," Global and Planetary Change, vol. 29, no. 1-2, pp. 99-119, 2001.

[7] S. Ma, Z. Wen, and J. Chen, "Scenario analysis of sulfur dioxide emissions reduction potential in China's iron and steel industry," Journal of Industrial Ecology, vol. 16, no. 4, pp. 506-517, 2012.

[8] H. W. Yang and X. L. Hu, "Study on inventory system of sulfur dioxide emissions in china," International Journal of Environmental Protection and Policy, vol. 18, no. 1, pp. 10-15, 2004.

[9] S. J. Smith, R. Andres, E. Conception, and J. Lurz, "Historical Sulfur Dioxide Emissions 1850-2000: Methods and Results," Tech. Rep. PNNL-14537, Richland, WA, USA, 2004.

[10] C. Li, Q. Zhang, N. A. Krotkov et al., "Recent large reduction in sulfur dioxide emissions from Chinese power plants observed by the Ozone Monitoring Instrument," Geophysical Research Letters, vol. 37, no. 8, pp. 292-305, 2010.

[11] J. Hao, S. Wang, B. Liu, and K. He, "Designation of sulfur dioxide and acid rain pollution control zones and its impacts on energy industry in China," Journal of Chemical Engineering of Japan, vol. 34, no. 9, pp. 1108-1113, 2001.

[12] H. K. Mohajan, "Chinese sulphur dioxide emissions and local environment pollution," International Journal of Scientific Research in Knowledge, vol. 2, no. 6, pp. 265-276, 2014.

[13] W. Zhou and D. M. Zhang, "An improved metabolism grey model for predicting small samples with a singular datum and its application to sulfur dioxide emissions in China," Discrete Dynamics in Nature and Society, vol. 2016, Article ID 1045057, 11 pages, 2016.

[14] Y. Yildirim, M. Bayramoglu, and S. Hasiloglu, "Prediction of sulfur dioxide daily levels in the City of Zonguldak using an adaptive neuro-fuzzy based method," Fresenius Environmental Bulletin, vol. 12, no. 10, pp. 1173-1179, 2003.

[15] S. Hassanzadeh, F. Hosseinibalam, and R. Alizadeh, "Statistical models and time series forecasting of sulfur dioxide: A case study Tehran," Environmental Modeling \& Assessment, vol. 155, no. 1-4, pp. 149-155, 2009.

[16] B. Li, T. Gasser, P. Ciais et al., "The contribution of China's emissions to global climate forcing," Nature, vol. 531, no. 7594, pp. 357-361, 2016.

[17] J. Liu, D. L. Mauzerall, Q. Chen et al., "Air pollutant emissions from Chinese households: A major and underappreciated ambient pollution source," Proceedings of the National Acadamy of Sciences of the United States of America, vol. 113, no. 28, pp. 7756-7761, 2016.

[18] S. F. Liu, Y. Dang, Z. Fang et al., Grey System Theories and Its Applications, Science Press, Beijing, China, 2014. 
[19] W. Meng and B. Zeng, Study on Fractional Operators and Grey Model, Science Press, Beijing, China, 2015.

[20] J. L. Salmeron, "An autonomous FGCM-based system for surveillance assets coordination," The Journal of Grey System, vol. 28, no. 1, pp. 27-35, 2016.

[21] C.-J. Chang, W.-L. Dai, and C.-C. Chen, "A novel procedure for multimodel development using the grey silhouette coefficient for small-data-set forecasting," Journal of the Operational Research Society, vol. 66, no. 11, pp. 1887-1894, 2015.

[22] A. Özdemir and G. Özdagoglu, "Predicting product demand from small-sized data: grey models," Grey Systems: Theory and Application, vol. 7, no. 1, pp. 80-96, 2017.

[23] Y. R. Liu, Y. Hu, and M. L. Hou, "A fractional order grey prediction algorithm," Journal of Grey System, vol. 14, no. 4, pp. 139-144, 2011.

[24] L. Wu, S. Liu, L. Yao, S. Yan, and D. Liu, "Grey system model with the fractional order accumulation," Communications in Nonlinear Science and Numerical Simulation, vol. 18, no. 7, pp. 1775-1785, 2013.

[25] L. F. Wu, S. f. Liu, and L. G. Yao, "Discrete grey model based on fractional order accumulate," Systems Engineering Theory \& Practice, vol. 34, no. 7, pp. 1822-1827, 2014.

[26] X.-P. Xiao, H. Guo, and S.-H. Mao, "The modeling mechanism, extension and optimization of grey GM $(1,1)$ model," Applied Mathematical Modelling, vol. 38, no. 5-6, pp. 1896-1910, 2014.

[27] S. Mao, M. Gao, J. Wen, and X. Nie, "Generalized admissible region of class ratio for fractional accumulated GM(1,1) model," The Journal of Grey System, vol. 26, no. 3, pp. 55-68, 2014.

[28] J. F. Cheng, Fractional Difference Equation Theory, Xiamen University Press, Xiamen, China, 2011.

[29] J. F. Cheng and G. C. Wu, "The solution of fractional difference equations of order (2,q)," Acta Mathematica Sinica, vol. 55, no. 3, pp. 469-480, 2012.

[30] D. E. Goldberg, "Genetic algorithms in search, optimization, and machine learning," Choice Reviews Online, vol. 8, no. 7, pp. 2104-2116, 1989.

[31] T. X. Yao and S. F. Liu, "Characteristics and optimization of discrete $\operatorname{GM}(1,1)$ model," Systems Engineering Theory \& Practice, vol. 29, no. 3, pp. 142-148, 2009.

[32] B. Zeng and S. F. Liu, "Direct modeling approach of DGM(1, 1) with approximate non-homogeneous exponential sequence," Systems Engineering \& Theory Practice, vol. 31, no. 2, pp. 297301, 2011. 


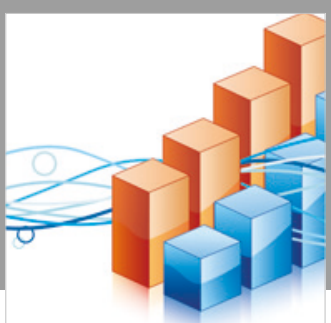

Advances in

Operations Research

\section{-n-m}
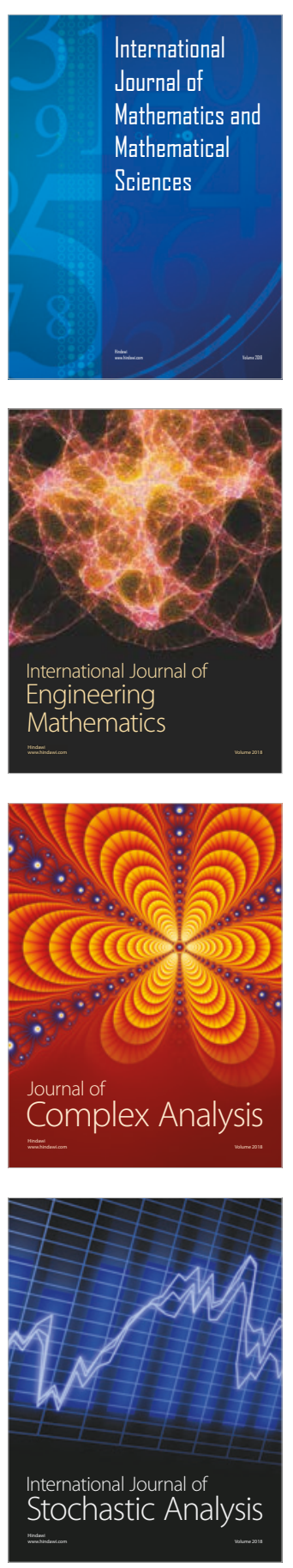
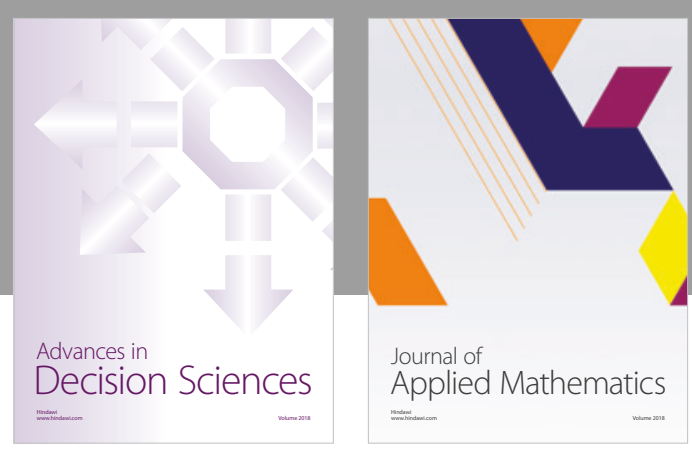

Journal of

Applied Mathematics
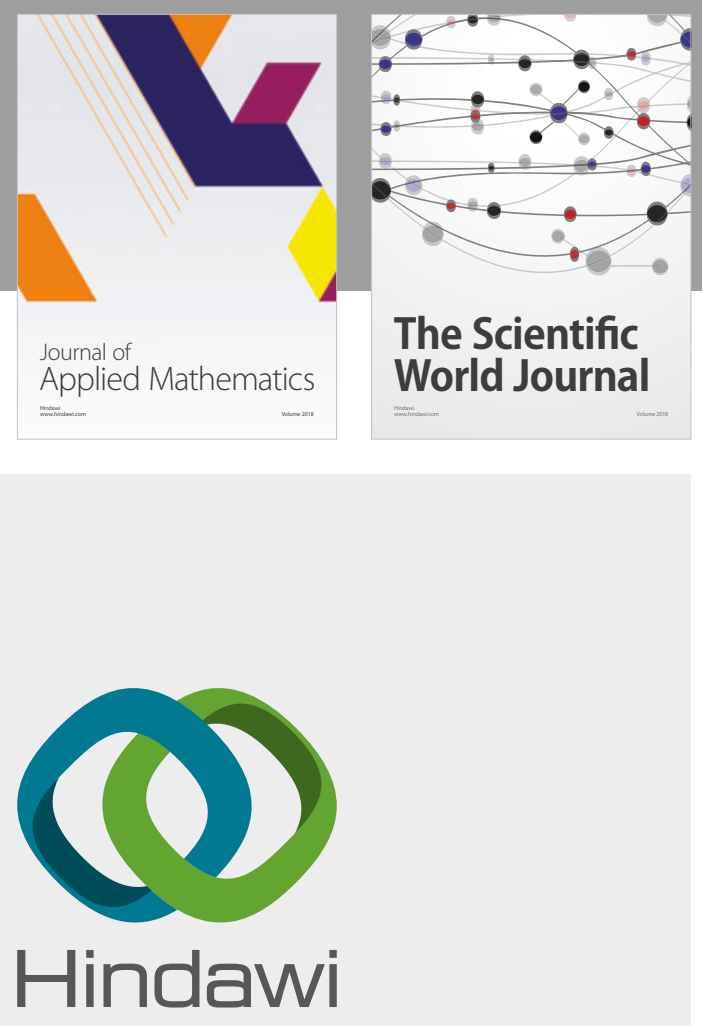

Submit your manuscripts at

www.hindawi.com

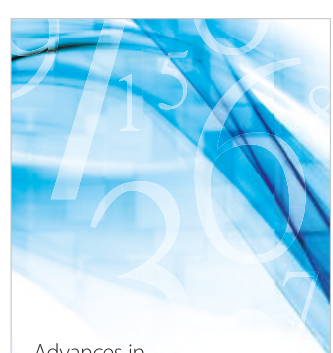

Advances in
Numerical Analysis
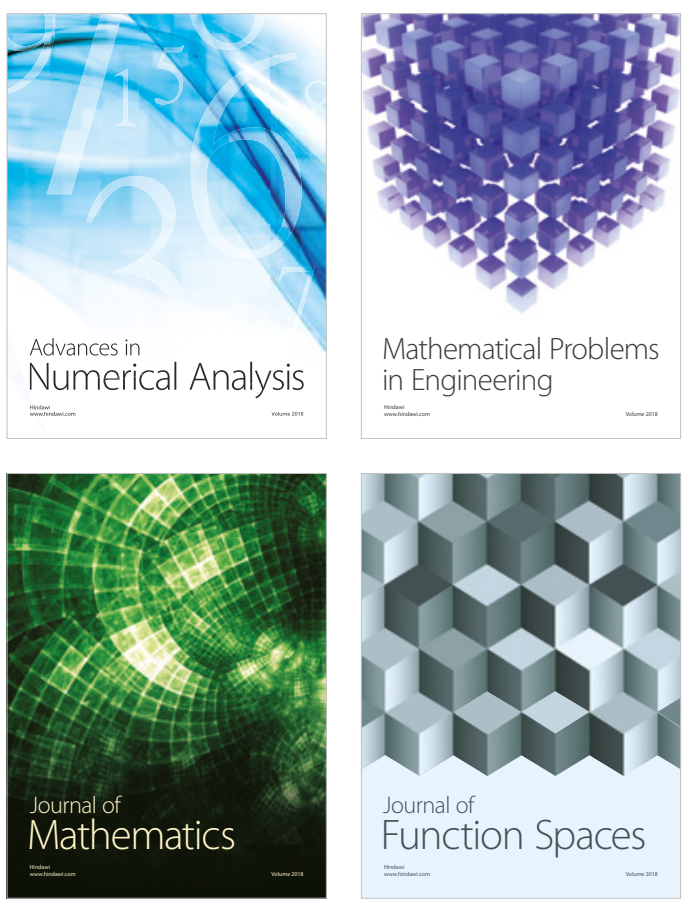

Mathematical Problems in Engineering

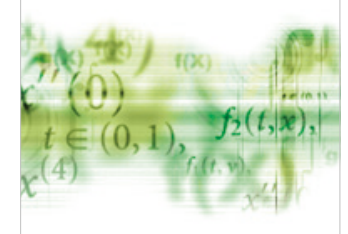

International Journal of

Differential Equations

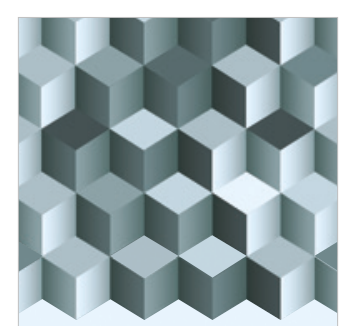

Journal of

Function Spaces
The Scientific

World Journal

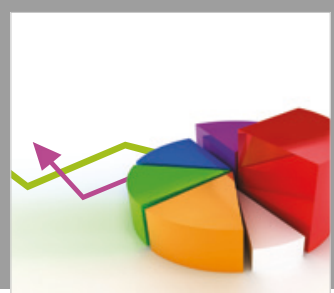

Journal of

Probability and Statistics
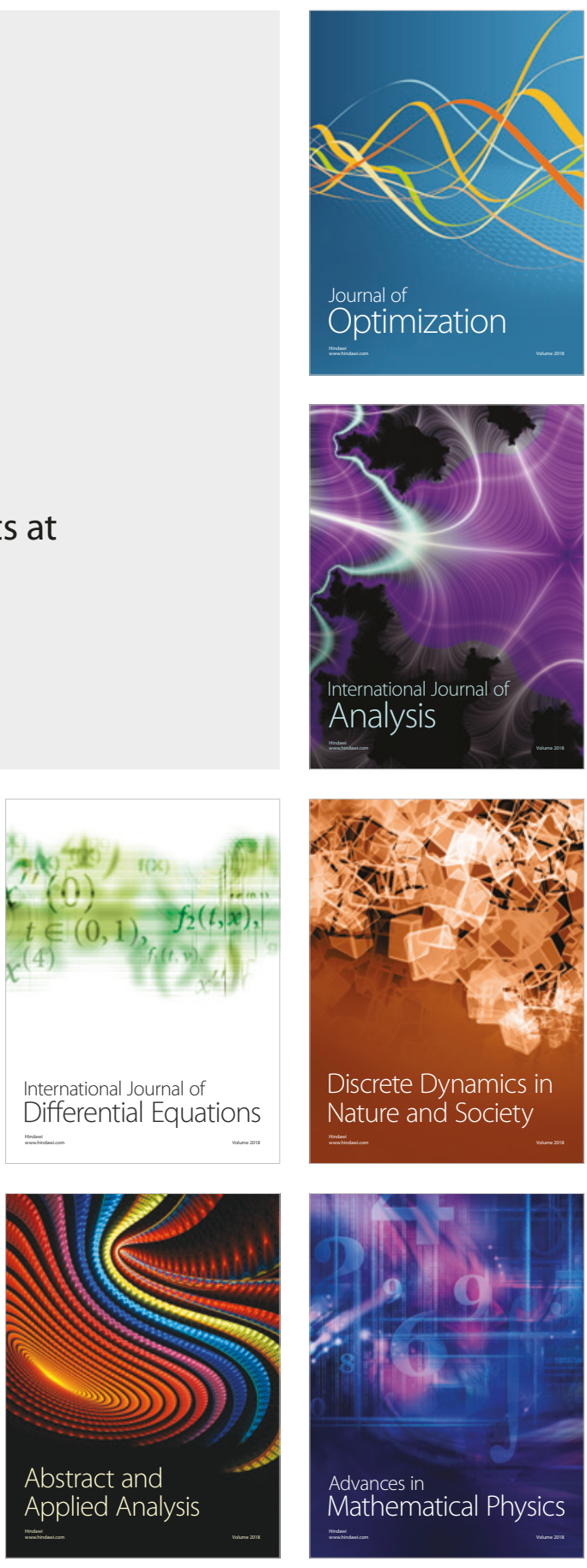